\title{
Nuevas rutas de la teología latinoamericana*
}

\author{
Diego Irarrázaval, \\ Instituto de Estudios Aymara, \\ Puno, Perú.
}

\section{Introducción: los caminos de la teología de la liberación}

"La teología de la liberación nació y sigue naciendo de la compasión con los que sufren y del compromiso con su marcha liberadora"l. Nacida en los años sesenta y guiada por el principio de la compasión, la teología latinoamericana y caribeña se ha llegado a constituir como obra sistemática según una novedosa metodología. En efecto, durante siglos la teología en América Latina ha asimilado modelos exógenos, coloniales, pero hoy contamos ya con un modo propio de proceder, basado en la espiritualidad compartida y en el trabajo de comunidades y de especialistas en todo el continente. Además, buscamos los contactos con los colegas de la teología asiática y africana y con pensadores del primer mundo, que reflexionan también desde la periferia.

Si nos preguntamos ahora cómo se hace teología, la respuesta depende de quién es el que la hace. En nuestro caso, se trata de una amplia gama de personas y grupos -en diversas situaciones socio-culturales- que creen tenazmente en la vida. La metodología latinoamericana - el cómo- está basada, por lo tanto, en una realidad plural, comunitaria, emancipatoria. Así, junto a ensayos sistemáticos están los trabajos hechos por la mujer, la población indígena, la comunidad negra. Pero lo importante es el denominador común a todo ello: se trata de comprender con fe la palabra y el espíritu. Nos acercamos, pues, a la revelación

- Este artículo es la ponencia, inédita en castcllano y ligeramente editada, presentada por el autor en una reunión de la Comisión Teológica de ASETT, en Nueva York, junio de 1994.

1. Clodovis Boff, "Epistemología y método de la teología de liberación", Mysterium Liberationis, I, Madrid, 1990, p. 89. 
del Dios vivo desde diversos puntos de vista: la racionalidad moderna, el ecofeminismo, el pensamiento oral y autóctono, las sabidurías cósmicas y sincréticas, pero lo fundamental es que los pueblos latinoamericanos y caribeños, enmarcados en cl sistema neo-colonial, confían, trabajan y rezan para lograr ser libres.

Tenemos, pues, una búsqueda común, que, como dicen las comunidades de base de Brasil, es una caminhada. Toda la vida, y también nuestro hablar de Dios, es un caminar. Para comprender la fe hay que ir de viaje, con una actilud de asombro, hacia la vida en abundancia. En términos de método, interpretamos la fe con los criterios de un pueblo pobre que anhela paz y libertad. Esta es la hermenéutica de un pueblo pobre y sabio, pues los "últimos" buscan siempre lo primordial: la salvación. A este caminar del pueblo nos sumamos teólogos y té́logas, y aportamos nuestros talentos.

En este continente sentimos también el llamado a ser, a la vez, católicos y ecuménicos. Lo católico se da en las inculturaciones concretas de la buena nueva universal de Cristo. Pero hemos comenzado a dialogar con otras tradiciones cristianas y no-cristianas, y ello en virtud de nuestra propia identidad. Como dice el concilio, la teología católica investiga "a la luz de la fe loda la verdad contenida en el misterio de Cristo" (Dei Verbum 24). Dios ofrece la verdad a todos los seres humanos, y nos acercamos a ella desde una gama de paradigmas culturales y religiosos, es decir, ecuménicamente. $\mathrm{E}$ insistimos en ello porque a menudo olvidamos el hecho de que el Espíritu Santo "no afecta únicamente a los individuos, sino también a la sociedad, a la historia, a los pueblos, a las culturas y a las religiones" (Juan Pablo II, Redemptoris Missio 28). Una teología fiel al Espíritu no puede aceptar las segregaciones actuales, económicas, culturales, eclesiásticas, religiosas, raciales, ni cualquier otra forma de comportamiento no ecuménico.

En esto está, creemos, lo más específico de la teología de la liberación, pero a ello hay que añadir las preocupaciones actuales que expresan nuestros colegas sobre la metodología de la teología latinoamericana2. Y en este sentido, quisiera reafirmar ante todo que compartimos la perplejidad y la inquietud con respecto al mundo de hoy. Por una parte, el pensamiento cristiano está siendo desafiado por nuevas rcalidades - la distancia creciente entre los pudientes y las multitudes excluidas - y está cambiando el paradigma de la modernidad; y por otra parte, vemos cómo sectores marginados asumen un protagonismo que no lenían hasta ahora. Lo que más nos preocupa, sin embargo, es el proyecto humano que se está gestando, pues queremos articular nuestra teología con

2. Me remito a conversaciones tenidas en Chile con Raúl Rosales, Fernando Castillo. Isabel Donoso, té́logos laicos, y con Sergio Torres, uno de los fundadores de ASETT; y en el Perú con Gustavo Guliérrez, Adelaida Sueiro y Emesto Cavassa. 
alternativas de vida más humana, y por ello nos cuestionamos qué paradigma subyace hoy a la teología de liberación.

¿Qué es hoy lo más esencial de esta teologla? Lo esencial no consiste en un conjunto de ideas, sino que sigue siendo la fidelidad cristiana, la praxis del amor. Nuestro modo de hacer teologfa brota de la fe, y por eso los grandes desafíos de hoy son, ante todo, que todo el pueblo de Dios asuma su responsabilidad teológica, el ecumenismo -en una época plagada de fundamentalismos-, el diálogo de la teología con la filosofía, la antropología, la psicología. Estamos, pues, ante una doble tarea. Por una parte, reafirmar nuestro punto de partida: el pueblo pobre como sujeto histórico, eclesial y teológico; y, por otra, ahondar en los nuevos retos: las culturas, la ecología, el pensamiento de la mujer, el reto de la modernidad, la búsqueda de alternativas económicas y globales.

A continuación vamos a analizar tres elementos que configuran nuestra metodología en la actualidad: los fundamentos de la teología latinoamericana, las exigencias actuales y el diálogo enıre las diversas teologras.

\section{Fundamentos de la teología latinoamericana}

Toda teologla cristiana tiene sus fuentes inagotables en los modos de comunicación de Dios a la humanidad - a través de la palabra y el Espíritu- y en las elaboraciones humanas sobre esa comunión a través de la sabiduría, el arte, la espiritualidad, la lucha por la vida, el gozo y la celebración. Estas elaboraciones son las respuestas de los seres humanos a la revelación divina.

\subsection{La palabra y el Espíritu}

La revelación a través de Cristo y su Espíritu es acogida por toda la comunidad creyente, cosa que hay recalcar: es todo el pueblo de Dios el que comprende y celebra la palabra y el Espíritu. El pueblo creyente lee los signos de los tiempos con los criterios del amor dados por el Espíritu. Por lo tanto, es en el seno del pueblo de Dios donde los teólogos y téologas examinamos la revelación.

Esta comunión en el Espíritu implica que la teología es ecuménica, es decir, expresa la exigencia a que se den lazos de unidad entre las denominaciones cristianas, que se dé el diálogo entre las diversas religiones y que se dé la apertura a posturas seculares (mal llamadas "no-creyentes") en nuestro continente. Esto es necesario porque cuando alguien es cristiano de forma sectaria no puede apreciar y comprender el amor de Dios presente en nuestra realidad multicultural y plurirreligiosa.

Estas fuentes - palabra, Espíritu- son las que alimentan la teología de la liberación, tanto en su versión pastoral como académica, y desde ahl el pueblo comprende la fe. 


\subsection{La sabiduría del pueblo}

En el continente latinoamericano hay numerosos elementos simbólicos y conceptuales con los que se hace teologia. En las numerosas sabidurias de los pueblos resalta la "relacionalidad", es decir, la comprensión de la realidad a partir de relaciones. La teología no piensa, pues, en términos de sujeto (que cree y piensa) y objeto (conceptualización de la fe). Trata de ver, más bien, cómo se relacionan entre sf todos los seres vivos.

Esta perspectiva lleva necesariamente a prestar gran atención a la realidad cotidiana. De esta manera el pensamiento cristiano brota del dolor y del hambre, de la solidaridad y de la esperanza; es decir, nace de la profundidad de lo cotidiano. Es, pues, una perspectiva de quienes caminan, con respeto y admiración, hacia el misterio.

A] mismo tiempo, todo ser humano es atraído por los ídolos contemporáneos y se aparta de la presencia de Dios. Por consiguiente, la teología tiene también una mirada crítica hacia las palabras y los símbolos populares. Hay que distinguir la oscuridad de la luz.

\subsection{El arte y la espiritualidad}

La belleza, la espiritualidad y la inteligencia de los pueblos se expresan a través del mito y del rito, del sincretismo y de la narrativa oral. Tanto el catolicismo popular y las comunidades evangélicas, como otras formas de religiosidad, constituyen tesoros de comunión con lo sagrado y de sus representaciones simbólicas. La realidad transcendente es comprendida a través de lo corporal, de la solidaridad con la gente postergada, de la música y la danza, del compartir alimentos y de tantos otros ritos cotidianos.

A las raíces de la teología latinoamericana que desde el inicio han sido la espiritualidad bíblica y la fe de las comunidades de base, añadimos ahora estos nuevos recursos: el arte y la contemplación de la gente sencilla, el canto, la peregrinación, las tradiciones del pueblo y muchos otros. Son recursos que alimentan una nueva forma holística de pensar la fe y nos conducen hacia la acción de gracias.

\subsection{La lucha por la vida}

La liberación es una preocupación diaria que afecta a lodo lo que somos. Hace unos años se insistía en los cambios económicos y políticos; hoy recalcamos el sentido integral de la liberación. Esto implica organización popular, resistencia cultural, sanación del cuerpo y del alma, tareas ecológicas, comunión espiritual, todo lo cual llega a formar parte del discurso teológico.

En comparación con la situación de hace unos años, no cabe duda de que 
nos encontramos en una grave crisis de modelos políticos y culturales. Pero los ojos de la fe están atentos a nuevos movimientos sociales urbano-populares, indigenas, afroamericanos, organismos de mujeres y de jóvenes. Abundan las iniciativas locales para luchar contra el empobrecimiento y la alienación, todo lo cual es discernido como signos, pequeños, pero fecundos, de una historia de salvación. Vemos también que las culturas originarias de nuestro continente, la sabiduria de la mujer, los nuevos movimientos de espiritualidad —cada uno a su modo- están afianzando una nueva humanidad y sanas relaciones con la totalidad del cosmos.

\subsection{El gozo y la celebración}

En medio del malestar y de la opresión radical, la gente del pueblo siente el consuelo de las bienaventuranzas. La risa y la fiesta transforman la realidad. Hay una comprensión dialéctica de la muerte y de la vida, del sufrimiento y de la alegría.

El pensamiento hegemónico exalta lo privado e individual, el éxito económico y tecnológico. Sin embargo, el corazón latinoamericano palpita a otro ritmo. Para nosotros, lo principal es compartir la fiesta, ser fieles a Dios, resolver nuestras contradicciones. Es decir, entendemos la liberación como celebración de la vida y no como mero cambio de unas estructuras por otras.

Estos fundamentos, la palabra, el Espíritu y nuestras elaboraciones de sabiduría, arte, espiritualidad, lucha y fiesta, constituyen los elementos de la teología de la vida cotidiana, desarrollada por las comunidades de fe. Vale decir, entonces, que la teología de la liberación no surge sólo de las cabezas de unos especialistas. Se trata más bien de un esfuerzo de colaboración en el seno del pueblo de Dios, donde abunda la sabiduría cordial. Los aportes básicos provienen de mujeres y hombres sabios y de las comunidades eclesiales.

Estas comunidades generan una teologfa holística, en la medida en que asumen el saber popular, su arte y su mística, su lucha por la vida y sus celebraciones. Y, a la inversa, cuando las comunidades de base se encierran en asuntos puramente intra-eclesiales, o en el puro pragmatismo social, o en la pura efervescencia carismática, entonces su comprensión y vivencia de la fe es muy parcial.

Hay que subrayar, por último, que esta teología concreta y holística está creciendo gracias al modo de pensar de la mujer, su modo de construir la vida, su espiritualidad y su celebración. Ella renueva y replantea la teología de la liberación en América Latina.

\section{Metodologías académicas}

Como ya hemos indicado, existe una sabiduría creyente del pueblo, una re- 
flexión hecha por personas con responsabilidad eclesial ("teología pastoral") y una elaboración sistemálica ("teología acadénica"). Aunque a ésta última no se la puede aislar de las ouras dos formas de pensar, vamos a analizar ahora la teología académica, sus opciones y modo de proceder metodológico, sin detenernos en sus mediaciones sociales y filosóficas, sus desarrollos doctrinales y sus aspectos pedagógicos y pastorales.

Durante tres décadas la teología latinoamericana ha trabajado creativamente, pero sin contentarse con sus logros iniciales ni estancarse en un determinado momento. El trabajo profesional académico de la leología de la liberación avanza en la actualidad, sin aferrarse a los logros iniciales ni estancarse en el camino. En este contexto será bueno recordar que lo característico de su metodologfa no ha consistido, ni menos aún se ha agotado, en pensar la revolución social desde la fe, y nos sorprende que así piensen algunos colegas del tercer mundo, como lo hacen sectores conservadores en el continente. Dicho esto, conviene revisar cuidadosamente lo que se ha avanzado a lo largo de estos treinta años y las nuevas vías - vamos a mencionar cinco- por las que transista esta teología.

Ante todo, la teología latinoamericana y caribeña se caracteriza por ser una intelección del amor liberador, un intellectus amoris. Como toda teología, es una reflexión desde la fe y orientada por la vida y enseñanza de la Iglesia. Su aporte especifico al pensamiento contemporáneo consiste en que reflexionamos pero no sólo con la mente, sino, por así decirlo, con el corazón. Nuestra teologfa está enraizada en el corazón y en la sabiduría del pueblo pobre a quien Dios ha escogido como portador del reino. Es, pues, algo distinto al pensar occidental predominante, analílico y pragmálico, sin que el amor forme parte de su mélodo. Esta innovación metodológica se debe al nuevo modo de ser teólogos y téblogas en la realidad latinoamericana: comprometidos y compasivos con la causa del pueblo que busca la vida. Gracias a este modo de ser y de compartir se dan avances metodológicos. Veamos ahora, brevemente, las cinco vías de la reflexión leológica.

\subsection{La teología sistemática}

Las tareas docentes, en una variedad de programas de la Iglesia y a veces en centros académicos, permiten y exigen una presentación sistemática de la fe desde la óplica del pobre. En esto han sido pioneras las obras de Juan Luis Segundo, Leonardo Boff, José Comblin; y desde la década de los ochenta existe una asociación que publica una colección de texlos de teología de la liberación. Los fundamentos fueron planteados desde el comienzo por Gustavo Gutiérrez, y éste añadió a la comprensión clásica de la teología como sabiduría espiritual y como saber racional, el que sea también "reflexión crítica de la praxis a la luz de la fe"3.

3. G. Gutiérrez, Teología de la liberación, Lima, 1971, pp. 16-34; La fuerza histórica de los pobres, Lima, 1979, pp. 103-108. 
Desde un punto de vista metodológico podemos esquematizar los tres momentos de este nuevo modo de proceder de la siguiente manera4:

a) lo pre-teológico: contemplación y acción, por amor al Cristo sufriente y resucitado.

b) la rellexión: consideración crítica de nuestra realidad, a través de "las mediaciones socio-analíticas", para establecer dónde hay muerte y dónde hay vida, y la reflexión desde la fe, como "principio hermenéutico determinante" (Instrucción Libertatis Nuntius X, 2). Los textos bíblicos y el magisterio eclesial son asumidos junto con la fe del pueblo de Dios, y es una reflexión que nos abre al misterio.

c) el producto teológico: en tomo a la praxis del amor, conversión, renovación eclesial y transformación de la historia, lo que implica formular metas inmediatas y estratégicas, análisis social, ética, programas de acción, etc.

Este esquema no ofrece un mecanismo rígido. Lo que hace es reafirmar el vínculo clásico entre espiritualidad, pensamiento y acción. Además, es también un esquema que subyace a otras metodologías más novedosas: hermenéutica del pueblo, teología de la mujer, teología afro-americana e indígena. $Y$ a su vez, el surgimiento y crecimiento de estas cuatro nuevas vías contribuyen a replantear toda la teología de la liberación. Esta no puede ser considerada como un edifício acabado, sino, siguiendo la metáfora, como la construcción permanente de una diversidad de hogares. Esto es lo que hace el pueblo de Dios, pueblo pobre y sabio que busca y festeja la libertad.

\subsection{La hermenéutica del pueblo}

Hay un modo latinoamericano de acoger, interpretar y poner en práclica la palabra, que forma parte de la sistematización recién expuesta, pero que tiene su propia dinámica y método. Muchos hoy leen regularmente la Biblia con apoyo de líderes laicos, especialmente mujeres, pastores-té́logos y exégetas, lo cual es considerado como una importante renovación de las bases de la Iglesia. Durante este siglo se han dado grandes pasos teológicos en esa dirección en las comunidades evangélicas, y, en los últimos veinticinco años, también en grupos católicos.

Este proceso ha sido animado y explicado por Carlos Mesters5. Al leer la

4. Esquema propuesto por Leonardo y Clodovis Boff, Como Fazer teología de Libertaçao?, Petrópolis, 1986, pp. 14-21, 37-63.

5. La manera en que la gente entiende la vida gracias a la Biblia es explicada por Carlos Mesters en "Lectura popular de la Biblia", en Breve diccionario reológico larinoamericano, Santiago, 1992, pp. 157-173. Por lo que toca a la nueva exégesis latinoamericana, véase Revista de interpretación biblica latinoamericana y Bibliografia brblica latinoamericana, ambas dirigidas por Milton Schwantes. Serfa bueno comparar y

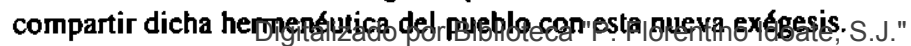

Universidad Centroamericana José Simeón Cañas 
Biblia, el pueblo examina su vida y descubre el poder para transformarla. Su lectura de la palabra no tiene como objetivo llegar a una interpretación de un texto sagrado, sino comprender su propia vida con la ayuda de la Biblia, y este tipo de lectura es el que permite al pueblo sentir a Dios como liberador. De esa forma, el "sentido" de la Biblia no consiste sólo en ser un mensaje para la mente humana, sino en ser ante todo consuelo y fuerza sentida con el corazón y puesta en práctica con las obras de la salvación.

Este método de lectura y vivencia de la palabra que ha descubierto el pueblo lo describe Mesters de esta forma:

a) un descubrimiento por parte de personas que narran lo que viven y comparan acontecimientos (sin obsesión de consumir conocimientos), y un acercamiento orante a la palabra. Se lee y se dialoga, se canta y se reza. Se descubre el "sentido" de la Biblia mediante el poder de la oración, en fidelidad al Espiritu.

b) una actividad comunitaria, ya que todos participan en la lectura de la palabra de acuerdo a los talentos de cada uno y a la maduración de su fe.

c) una interpretación de la Biblia, que forma parte de un proceso más amplio. En primer lugar, se analiza crfticamente la propia realidad y se llega a comprender los problemas de cada dia con la ayuda de las ciencias humanas. En segundo lugar, se ubican las experiencias cotidianas al interior del proyecto salvífico de Dios. Se estudia la realidad b́blica y también la realidad de quienes hoy leen la Biblia. Gracias a esta lectura, la cruz, que es signo de muerte, pasa a ser también una señal de resurrección. Por último, se comparte comunitariamente. En un ambiente de confianza y de fe, de diálogo y de carin̄o, se deja que el Esplritu muestre la presencia de Cristo.

La comunidad es, por lo tanto, la que descubre la relación entre la Biblia y la vida de hoy. Se trata de una interpretación hondamente humana, y en este sentido, es también ecuménica, ya que defiende la vida en contextos de muerte. También saca a luz la manipulación que algunos hacen de la Biblia desde la perspectiva de los Ídolos contemporáneos. Y en sentido positivo, la lectura popular y orante invila a la comunidad a continuar luchando por la vida y a cultivar la esperanza.

\subsection{La teología de la mujer}

En esta teología no se trata simplemente de incorporar el "tema" de la mujer, ni de feminizar la teologfa. Se trata, más bien, de adoptar una perspectiva holística, que proviene de vivencias de mujeres y varones, y que desde ahí examina la realidad cristiana y también de otras tradiciones religiosas. Por un lado, cuestiona la opresión patriarcal en la sociedad, la Iglesia y la teologia, y, por otro, reflexiona sobre las relaciones humanas y la apertura al misterio de la vida. 
Según Ana María Tepedino y M. Ribeiro6, los puntos de partida de esta teología son las experiencias de la mujer, su fe en Dios y una determinada praxis de relaciones humanas. Combina el rigor cientfico, haciendo uso de las ciencias, con la sensibilidad humana. Con criterios biblicos interpreta tanto la opresión como las fuerzas de la vida. Relee la revelación y también la realidad de cada dla con vistas a una liberación. Elsa Tamez y otras teólogas leen la palabra desde la perspectiva de género, de las culturas y de los procesos sociales.

Otro aporte relacionado es el eco-feminismo7. Brota del dolor, la lucha y la espiritualidad de las mujeres, en especial de las empobrecidas. Puede analizar y confrontar el patriarcado, porque cuenta con una antropologla y una espiritualidad distintas. Concibe lo humano en articulación con todo lo viviente, y la transcendencia es considerada como la relación que une a todos los seres del universo. Esta teología dialoga no tanto con las ciencias sociales, sino especialmente con una antropología unitaria e igualitaria de la tierra y del cosmos. Además, critica el patriarcalismo presente en la dogmática cristiana y también en la teología de la liberación.

Como aporte específico, hace una lectura alternativa de la tradición cristiana. Relee teologicamente las historias de mujeres, los ritos y símbolos, el arte, el lenguaje corporal, la organización que acrecienta la vida, es decir, lo cotidiano que es camino al reino y apertura al misterio. La mujer siente a Dios de otro modo, y por ello habla de Dios, de Jesús y de María con su propia simbología. Como ha dicho Ivone Gebara, en el próximo milenio la categoría de género dará un aporte revolucionario a la epistemología cristiana, ya que el género produce una nueva comprensión de la condición humana.

\subsection{Teología afro-americana}

Esta teologfa se desarrolla a partir de profundas raices en la historia y sabiduría de poblaciones negras, principalmente en Brasil, el Caribe, Panamá, Ecuador y Colombia. De forma similar a la teologla de la mujer y de los pueblos indígenas, también la teología negra surge de un pensar comunitario, con identi-

6. Cfr. Ana Marfa Tepedino y Margarita Ribeiro, "Mujer latinoamericana", en Vida, clamor y esperanza, Bogotá, 1992, pp. 379-388; El rostro femenino de la teologia, San José, 1986; Aportes para una teología feminista, Santiago de Chile, 1990.

7. Ivone Gebara, "Presencia de lo femenino", en VV. AA., Cambio social y pensamien10 cristiano en América Latina, Madrid, 1993, Pp. 199-214. En el encuentro de ASETT, en Nueva York (1994), Ivone nos ponía en guardia de "no reducir lo metodológico a un tipo de discurso racional que puede ser manipulado" por la academia y por el racionalismo occidental. "Por ejemplo, sacerdotisas en la religión brasileraafricana tienen una metodologfa, un modo de hacer su propia teologfa... Es una tradición oral como todas las culturas de pueblos pobres". 
dad propia y con alternativas humanas, y está relacionada con tradiciones religiosas (algunas cristianas y otras no-cristianas). Tiene claras opciones y metodologías8. Esta teología es hecha por la comunidad negra, presente en un amplio movimiento social, que tiene identidad y espiritualidad afro-americanas y que combate la esclavitud y el racismo.

Marcos Rodríguez asume el método global de la teología de la liberación, pero le añade rasgos específicos, pues el pueblo negro liene su modo de pensar y de actuar, y comprende a Dios, a Jesús y a la comunidad a partir de prácticas religiosas africanas. José $\mathrm{G}$. Rocha pone de relieve la epistemología de autoafirmación como personas negras (en contraste con la ideología dominante antinegra), el rescate de la memoria histórica de la esclavitud y de los movimientos socio-culturales, y la existencia de um Deus diferente que nos fortifica enquanto negras e negros, e que este Deus necesita ser por nós explicitado. Antonio A. da Silva afirma que "somos impulsados por el Esprritu Santo a encontrar a Cristo en las culturas... y en el pueblo negro... en su pasión y resurrección". En los próximos años, el modo de hacer teología negra podrá fortalecer el diálogo con las religiones africanas y también con los movimientos que luchan por superar la pobreza.

\subsection{La teología de los pueblos indígenas}

Durante más de veinte siglos, los pueblos originarios de Abya Yala han compartido sus cosmologías y su fe, y ahora, desde hace unos años, están teniendo una serie de diálogos a nivel local y a nivel continental9. En este proceso se está elaborando y afianzando una melodología.

Corno dice Eleazar López, la teología india "acompaña, explica y guía el caminar de nuestros pueblos indios a través de toda su historia". Su rafz es "la fe en el Dios de la vida", "resultado de la revelación de su amor y de sus designios en el devenir de la historia y culturas de nuestros pueblos". Eleazar señala las siguientes características de esta teología india:

8. Cfr. Marcos Rodríguez da Silva, Teología afro-latinoamericana, Quito, 1990; José G. Rocha, "Reflexión teológica, en Agentes de Pastoral Negra, Sao Paulo, 1993, pp. 63-69; Antonio A. da Silva, "Evangelización liberadora a partir de los negros", en Vida, clamor, esperanza, Bogolá, 1992, pp. 425-433; VV. A.A., Cultura negra y reología, San José, 1986.

9. Cfr. Eleazar Lopez, "Teología india hoy" en Primer encuentro latinoamericano, teologia india, México, 1990, pp. 5-16, y Caminar teológico de los pueblos indios, 1992; Clodomiro Siller "Anotaciones metodologicas", y Petul Cut Chab "Conceptos básicos de la teología india", en Encuentro-diálogo sobre metodología teológica latinoamericana, México, 1993, y "Experiencia de Dios en los proyectos de vida de los pueblos" en Segundo encuentro latinoamericano, Panamá, 1993. 
a) una teología sumamente concreta, que contempla la vida, la saborea y se baña en sus misterios, que acompaña proyectos de vida de nuestros pueblos.

b) una comprensión holística de la existencia del pueblo y de lo sagrado.

c) un lenguaje mítico-simbólico que expresa el sentido profundo de la vida (y no un arcaísmo pre-moderno), es decir, una preferencia por el lenguaje religioso y ritual.

d) el sujeto de la teología es el pueblo que elabora su pensamiento de forma colectiva, es decir, sabias y sabios en las comunidades y pueblos indígenas.

El diálogo gira en tomo a la tierra, la organización, los ritos religiosos, los esfuerzos para vivir en libertad. Todo esto es reflexionado en celebraciones, urabajo en grupos, diálogos amplios, ponencias, charlas y acuerdos a través del consenso. Si lo comparamos con otras maneras de hacer teología, aqui abundan y se disfrutan momentos de oración y fiesta.

La teología india tiene como matriz la vida real de los pueblos (racialmente discriminados y empobrecidos, pero con identidades y comunidades recias). Sus sujetos son sabias y sabios, comunidades, pueblos. Sus fuentes son la madretierra y la cosmovisión que la acompaña, mitos, ritos y símbolos, y la Biblia, que es "espejo para comprender la presencia de Dios en nuestra historia, cultura y religión", como dijeron en el diálogo de México, en 1993. Su finalidad es responder a necesidades vitales de pueblos originarios y transformar la realidad en vistas al reino. En la teología india hay una epistemología relacional, siempre masculina y femenina, concreta, simbólica, corporal, ritual, contemplativa. Tiene pues su propia lógica y sabiduría. Ha establecido vínculos con la teología de la mujer, la teología negra y la perspectiva sistemática, ya que hay sensibilidades, preocupaciones y metas comunes.

\subsection{Otras metodologías}

Además de las que acabamos de exponer, existen otras metodologías. Durante varias décadas, historiadores y teólogos están analizando los movimientos socio-culturales "desde abajo", y asf también lo hacen con las estructuras eclesiales en América Latina y el Caribe 10, mientras otros comienzan a relacionar la ecología con la teologíalı. Pero también hay expresiones latinoamericanas

10. Enrique Dussel, Eduardo Hoomaert, Pablo Richard, Max Salinas y otros han dirigido volúmenes de historia de la Iglesia desde la óptica de la liberación. Ver también Cehila, Historia de la seología en América Latina, San José, 1980; Roberto Oliveros, Liberación y reología Génesis y crecimienıo de una reflexión, 1966-1977. Lima, 1977. Cehila, Raíces de la teología latinoamericana, San José, 1985.

11. Cfr. V. Serrano (ed). Teología de la ecología, Quito, 1991; Marcelo de Barros y J. 
de las teologías dominantes, con el reduccionismo metodológico de convertirse prácticamente sólo en hermenéutica del magisterio, según pautas filosoficas occidentales -cosa que no vamos a analizar ahora.

Para terminar, quisiera resumir los logros y los desafíos compartidos por las principales corrientes mencionadas: la sistemálica, la hermenéutica del pueblo, la teología de la mujer, la teología negra y la teología india. Todas estas teologías - debido a la fe común que las subyace - son solidarias con una humanidad sufriente y también festiva, opción que las distingue de teologías centradas y preocupadas, casi exclusivamente, por analizar y explicar la doctrina cristiana. Dicha opción solidaria incluye una lectura crítica de la experiencia y realidad latinoamericana a fin de transformarla.

Eslos elementos fundamentales compartidos hacen que las cinco corrientes mencionadas fonnen como una "familia teológica" de teologfas hermanas y distintas. Tienen nombres distintos, pero los apellidos son los mismos, y lo que interesa no son tanto sus denominaciones, sino su significado para el pueblo, que así conoce su fe y alianza su esperanza en medio de condiciones de muerte.

No cabe duda de que estamos dando grandes pasos metodológicos. La teología de la liberación avanza por nuevas rutas. Está siendo re-creada, o, como dice P. Richard, hay "caminos inéditos e insospechados para el crecimiento de la teología de la liberación"12. Hay nuevos temas y desaffos. La reflexión hoy está en manos de muchas comunidades de base, de la mujer, del pueblo afroamericano e indígena. Estos son los sujetos de la teología, gracias a su sabiduría.

Hoy también podemos y tenemos que seguir ahondando en la crítica al capitalismo sacrificial, al patriarcado, al racismo, al colonialismo cultural, y estos cuestionamientos dan color y sabor al discurso teológico. Hay que recalcar también la novedad y la pluralidad de la teología hecha por académicos. Y hay que mencionar, por último, la dimensión ecuménica, ya que, por una parte, tenemos la fe como contenido y principio hermenéutico, y, por otra, estamos en relación con otras tradiciones religiosas indígenas, afro-americanas, espiritualidades de la mujer.

\section{La interacción entre las diversas teologías}

Las distintas comprensiones de la fe nos ofrecen la posibilidad de una mayor comunicación y maduración teológicas. Voy a detenerme sólo en tres retos: los vínculos entre las fuentes del saber popular y la actividad de los teólogos profe-

L. Caravias, Teología de la tierra, Madrid, 1988; y el último libro de Leonardo Boff sobre ecología.

12. P. Richard, "La teologfa de la liberación en la nueva coyuntura", Pasos 34 (1991). 
sionales, la interacción entre las corrientes latinoamericanas ya anotadas y el diálogo con otros teólogos y teólogas del tercer mundo.

Esta interacción no es un mero intercambio de información, ni es solo un hablar sobre "otros" sin entrar en comunión con sus metodologías. Lo importante es escuchar otros modos de pensar y dejarse interpelar por ellos, y contribuir así a fortalecer al pueblo pobre que anhela su liberación. En mi opinión, las personas que nos dedicamos a la teologla de forma profesional poco o nada tomamos en cuenta los retos recién mencionados. Cada uno de nosotros tiende a moverse en su territorio y sus temas de especialización. El diálogo es poco sistemático y poco crítico, y por ello la interacción requiere una conversión de parte nuestra.

Veamos el primer reto. El pueblo de Dios responde a la palabra y al espíritu y desarrolla su sabiduría creyente. Tiene una epistemología de la "relacionalidad"; su arte y espiritualidad tienen contenidos teológicos; lucha también a favor de una liberación concreta, celebra fiestas y gracias a ellas ve a Dios como vida. La pregunta es si estas verdades forman parte de nuestra agenda teológica, si afectan el modo de pensar que nos caracteriza como profesionales. Y la respuesta es que no, aunque hay excepciones, pues algunos dialogan con el saber del pueblo. El gran reto es que los paradigmas latinoamericanos, la "relacionalidad" y la celebración que fundamentan el conocimiento, y la espiritualidad holística, penetren en el corazón de la teología sistemática. No se trata simplemente de tenerles en cuenta de alguna forma, ni de que estén presentes en las implicaciones pastorales, sino de sistematizar la fe a partir de la "hermenéutica del pueblo pobre y sabio".

Un segundo reto es tejer juntos una manta multicolor. Las cinco principales corrientes de reflexión que hemos mencionado no están entrelazadas. No hay un intercambio permanente que molive nuevos modos de pensar. La mayoría de nosotros desconoce las otras metodologías, con la excepción de la teolog ‘a indígena, que invita a representantes de otras teologías a sus congresos. La teología de la mujer parece ofrecer mejores condiciones de diálogo entre las distintas perspectivas. Pero, por otra parte, poco a poco, la teología de la liberación reconoce que hay sujetos teológicos con distintas epistemologlas, y que el encuentro entre todos ellos es fecundo. En cualquier caso, a todos se nos exige confrontarnos con los ídolos contemporáneos del patriarcado, el racismo, la negación de otras culturas, el dios-dinero. Dicho en forma alirmativa, cada coriente teológica contribuye en la búsqueda común de alıernativas.

El tercer gran reto es el encuentro con otras teologías del tercer mundo. Pocos pueden estar presentes en diálogos que se llevan a catio en otros continenles, pero todos pueden abrir el corazón y la mente a otros modos de hacer teología. La reflexión asiática nos lleva a ver la salvación de los pueblos de religiones no-cristianas, a comprender la calidad religiosa del pobre y a hacer 
teologia haciendo uso de conceptos no occidentales. Su crftica teológica contra la pobreza va de la mano con la propuesta de una nueva humanidad. Los colegas de Asia también nos sensibilizan a un diálogo de dimensiones cósmicas, soteriologías metacósmicas, el shamanismo y otras religiones del pueblo, nos plantean la relación entre espiritualidad y leología, y la apertura tanto a la palabra biblica como a textos sagrados no-cristianos.

Las teologías de Africa nos presentan ricos esquemas antropológicos y cosmológicos, y comparten con nosotros su desaflo de inculturación. También impacta la atención que prestan a la palabra de Dios y a las tradiciones religiosas; all germina una modalidad de ecumenismo. Al reflexionar en medio de condicionamientos coloniales y racistas, y combatirlos, nos motivan a los latinoamericanos a asumir estas realidades. También llama la atención su capacidad de hacer análisis socio-cultural y religioso en el quehacer teológico.

También nos enriquecemos con las reflexiones hechas por los sectores marginados en el primer mundo. La teología negra de Norteamérica ha favorecido el nacimiento de un pensar afro-latino-americano. Su cristología amplía nuestros horizontes y cuestiona categorías, sutil o abiertamente racistas, que han penetrado en el cristianismo. Su reflexión de fe es holística; toma en cuenta lo religioso, lo cultural, lo económico, lo político y el género. Como ha dicho James Cone, "la verdad se encuentra en las historias, culturas y religiones de nuestros pueblos". Esta visión holística también nos es comunicada por la teología hispano-latina de Norteamérica. Sus reflexiones sobre el mestizaje y sobre la religión popular, sobre la inculturación y sobre la lucha por un mundo nuevo, son impulsos que renuevan nuestra visión de fe.

El diálogo entre latinoamericanos y otros teólogos y teólogas del tercer mundo es aún débil y esporádico. A menudo nos ven como arrogantes, como si quisiéramos imponer nuestro modelo de teología de liberación a todos los demás. ¿Qué podemos hacer? Como sugiere Gustavo Gutiérrez, hay que escuchar y aprender a trabajar juntos. Al dialogar, "escuchamos lo que nos dicen las teologías hermanas" y a través de ellas escuchamos a pueblos que tienen "experiencias de fe expresadas teológicamente". Además, aprendemos a hablar de Dios. "Un nuevo lenguaje profético y místico con respecto a Dios ha brotado en estas tierras llenas de opresión y de esperanza. Aprendemos de nuevo a cómo decir "Dios"'3. Esta actitud de trabajo en conjunto vale también al interior de América Latina. Las diversas versiones de la teologfa de la liberación crecen mucho cuando interactúan y aprenden unas de otras. Estos diálogos no tienen

13. G. Gutiérrez, "Reflections from a latinoamerican perspective: finding our way to talk aboul God", en Virginia Fabella y Sergio Torres, Irruption of the third world; Nueva York, 1983 (libro clásico, que resume lo avanzado por las teologías del tercer mundo). 
una meta encerrada en sí misma. Lo fundamental es cómo los pueblos, sus té6logos y teólogas, caminan hacia el misterio

\section{EL camino hacia el misterio}

El método es un caminar que vale en la medida en que es respuesta de fe al misterio de Dios presente en nuestra historia Como ya he dicho, es la caminhada de fe del pueblo sabio. Se avanza en medio de muchos obstáculos, inseguridades, retrocesos, pero convencidos de que la muerte es transformada en vida.

La lectura biblica hecha por comunidades marginadas, en Africa, en América Latina, en comunidades negras e hispánicas de Norte América, fortalece la esperanza. Allí se entiende la condición humana en términos de la llegada del reino, el gozo del cielo para los hambrientos, la paz y la justicia del Señor, el coraje que proviene del Espíritu. En esta lectura bíblica hay un movimiento teológico hacia el Dios vivo.

También se siente el misterio al entrar en comunión con la gama de corrientes religiosas que hay en nuestro continente: religiones mestizas, espiritualidades de la mujer, religiones indigenas, religiones negras. Se siente y comprende la maldad dentro y en torno al ser humano. Se siente y se analiza la "gracia cotidiana": la salvación, los vínculos entre personas, el perdón y la compasión, la belleza de la tierra, la humanización de la política, los ritos de cada día. Así es como los pueblos y sus iglesias caminan hacia el misterio, que es lo que enmarca la labor teológica. El objetivo de nuestra reflexión no es, pues, saber más, sino comprender mejor las maravillas del vivir.

Desde sus inicios, hace treinta años, la teología latinoamericana de la liberación, metodológicamente, está enraizada en la espiritualidad. El pensamiento es inseparable de la mística de la lucha y del gozo de la celebración. Esta opción metodológica se ha fortalecido gracias a los contactos con teólogos y teólogas de Asia y Africa y con la reflexión de los pueblos indígenas en la dimensión espiritual de la tarea teológica. ¡No es una dimensión! El corazón de la teología es, más bien, admirar el misterio.

En la fe, las palabras son significativas cuando nacen de y llevan al silencio y a la contemplación. Por lo tanto, el misterio no es un asunto platónico de imágenes y arquetipos. Tampoco es un asunto de ritos misteriosos conocidos por una élite. Se trata, más bien, de la maravillosa acción divina en la historia que ha sido revelada a los últimos y a las últimas. En otras palabras, el misterio de Cristo —celebrado y reflexionado por nuestros pueblos pobres y sabios- es el centro de nuestro trabajo teológico, en el sentido de que peregrinamos hacia el misterio. 